\title{
Determining the origins of impurities during azide- alkyne click cyclization of polystyrene
}

\author{
Ravinder Elupula ${ }^{a}$, Joongsuk $\mathrm{Oh}^{b}$, Farihah M. Haque ${ }^{a}$,Taihyun Chang ${ }^{b}$, and Scott \\ M.Grayson ${ }^{a * \pm}$ \\ ${ }^{a}$ Department of Chemistry, Tulane University, New Orleans, 70118, USA \\ ${ }^{\mathrm{b}}$ Department of Chemistry and Division of Advanced Materials Science, Pohang University of \\ Science and Technology (POSTECH), Pohang, 37673, Republic of Korea
}

\section{$\underline{\text { Table of Contents }}$}

Synthetic Protocols

S2

Analytical Protocols

S3

Table S1: MALDI-TOF and SEC Molecular Weight Data

S4

Figure S1: HPLC Chromatograms of the Separation of Dimers from Unimers of $\boldsymbol{l}$-PS2800 and $c$-PS 2800

S5

Figure S2: HPLC Chromatograms of the Separation of Dimers from Unimers of $l$-PS 3700 and $c-\mathbf{P S}_{3700}$

Figure S3: MALDI-TOF mass spectra in reflector mode of $l-\mathbf{P S}_{2800}, c-\mathbf{P S}_{2800}, l d-\mathbf{P S}_{2800}$, and $c d-\mathbf{P S}_{2800}$

Figure S4: MALDI-TOF mass spectra of $l$-PS $3700, c-P_{3700}, l d-P_{3700}$, and $c d-$ PS $_{3700}$ S8 
Figure S5: MALDI-TOF mass spectra in reflector mode of $l$-PS ${ }_{3700,} c$-PS $3700, l d-$ PS $_{3700,} c d$ PS3700

Figure S6: Representative SEC Chromatograms of a) purified $l$-PS2800 and $l d$-PS2800 and of c) purified $c$-PS2800 and d) $d$-PS2800 after 30 days

S10

Figure S7: Representative SEC Chromatograms of a) purified $l$-PS 3700 and $l d$-PS 3700 and of c) purified $c$-PS3700 and d) $d$ - $\mathbf{P S}_{3700}$ after 30 days

Figure S8: SEC Chromatograms of the Azidization Reaction Generating l-PS2800, 2a at 0, $1,4,16$, and $48 \mathrm{~h}$

Figure S9: MALDI-TOF Mass Spectra of the Azidization Reaction Generating l-PS2800, 2a at 0 and $48 \mathrm{~h}$

\section{Synthetic Protocols:}

$\boldsymbol{\alpha}$-Ethynyl- $\omega$-azido-polystyrene, 2 . The $\alpha$-ethynyl- $\omega$-bromo-polystyrene $1(20 \mathrm{mg}, 0.0074$ mmol) (prepared according to Laurent and Grayson) $)^{1}$ was dissolved in dimethylformamide (1 mL). Sodium azide (48 $\mathrm{mg}, 0.74 \mathrm{mmol}$ ) was added to the reaction mixture, and then proceeded to stir under ambient conditions. Aliquots $(0.2 \mathrm{~mL})$ were taken at the specified time points and diluted with dichloromethane $(10 \mathrm{~mL})$, followed by washing with deionized water $(3 \mathrm{x} 5 \mathrm{~mL})$. The organic layer was collected and dried over anhydrous magnesium sulfate. After filtration, the solvent was removed in vacuo yielding $2-4 \mathrm{mg} /$ aliquots of a white powder ( $>50 \%$ yield).

Cyclic polystyrene, 3. The $\alpha$-ethynyl- $\omega$-azido-polystyrene $2(0.123 \mathrm{~g}, 0.0350 \mathrm{mmol})$ and 100 $\mathrm{mL}$ dichloromethane were added to a 2-neck round bottom flask. A solution of PMDETA (0.415 $\mathrm{g}, 2.4 \mathrm{mmol})$ in $\mathrm{CH}_{2} \mathrm{Cl}_{2}(150 \mathrm{~mL})$ was added to a second 2-neck round bottom flask. The flask containing the linear polymer, underwent 2 freeze/pump/thaw cycles. The flask containing the PMDETA solution, underwent 2 freeze/pump/thaw cycles. Upon an additional freeze, $\mathrm{CuBr}$ (0.315 $\mathrm{g}, 2.2 \mathrm{mmol}$ ) was added to the frozen solution of PMDETA in $\mathrm{CH}_{2} \mathrm{Cl}_{2}$. The flask was 
resealed, refilled with $\mathrm{N}_{2}$, pumped, and thawed. Upon both flasks reaching room temperature, a gastight syringe was filled with the polymer solution and added to the CuBr/PMDETA solution via a syringe pump at a rate $2.0 \mathrm{~mL} / \mathrm{min}$. Once the polymer was completely added to the $\mathrm{CuBr} / \mathrm{PMDETA}$ solution, the mixture was allowed to stir an additional 2 hours to ensure complete cyclization. The reaction mixture was opened to air, and washed with several portions of saturated aqueous ammonium chloride, until the organic and aqueous layer were colorless. The organic layer was collected and dried over anhydrous magnesium sulfate. After filtration, the solvent was removed in vacuo to yield $108 \mathrm{mg}$ of a white powder (87.8\% yield). .

\section{Analytical Protocols:}

Size-Exclusion Chromatography (SEC) data were acquired using two different setups. Data from Figure 1, Table 1, Figure 3, Figure S6, and Figure S7 are from a Bishoff HPLC compact pump with THF as the mobile phase with a $0.7 \mathrm{~mL} / \mathrm{min}$ flow rate with columns heated at a constant 40 ${ }^{\circ} \mathrm{C}$ by a column oven. This system was operated with a set of two PLgel Mixed-E columns (Agilent $300 \times 7.5 \mathrm{~mm}$ ) in series. Viscotek TDA 302 detector was used as refractive index detector. The instrument was calibrated with a set of polystyrene standards. Data from Figure S8 are from a Waters model 1515 isocratic pump (Milford, MA) with THF as the mobile phase with a $1 \mathrm{~mL} / \mathrm{min}$ flow rate with columns heated at a constant $30^{\circ} \mathrm{C}$ by a column oven. This system was operated with a set of two columns in series from Polymer Laboratories Inc. consisting of PSS SDV analytical linear M $(8 \mathrm{~mm} \times 300 \mathrm{~mm})$ and PSS SDV analytical $100 \AA(8 \times 300 \mathrm{~mm})$ columns. A Model 2487 differential refractometer detector was used as a refractive index detector. The instrument was calibrated with Polystyrene ReadyCal Standards from Waters.

For the normal phase liquid chromatography (NPLC) separation, a bare silica column (Nucleosil. $5 \mu \mathrm{m}, 50 \AA, 250$ x $4.6 \mathrm{~mm})$ was used. Mobile phase was a THF/n-hexane mixture (37/63, v/v) delivered by an HPLC pump (Shimadzu LC-20AD) at a flow of $0.5 \mathrm{~mL} / \mathrm{min}$. The column temperature was kept at $20^{\circ} \mathrm{C}$ using a homemade column jacket and a water bath circulator (Julabo F25). Chromatograms were recorded with a UV/Vis absorption detector (YounlinUV730D). All samples for NPLC analysis were dissolved in the eluent at a concentration of $1 \mathrm{mg} / \mathrm{mL}$ and the injection volume was $100 \mu \mathrm{L}$. 
Mass spectral data were collected using two different instruments. Data in Table 1, Figures S3, S5, and S9 were collected from a Bruker-Daltonics Matrix Assisted Laser Desorption Ionization Time-of-Flight (MALDI-TOF) Autoflex III mass spectrometer in reflector mode with positive ion detection. Typical sample preparation for MALDI-TOF MS data was performed by making stock solutions in THF of matrix $(20 \mathrm{mg} / \mathrm{ml})$, polymer analyte $(2 \mathrm{mg} / \mathrm{ml})$, and an appropriate cation source $(2 \mathrm{mg} / \mathrm{ml})$. The stock solutions were mixed in a 20/5/2 ratio (matrix/analyte/cation), deposited onto the MALDI target plate and allowed to evaporate via the dried droplet method. For polystyrene derivatives, dithranol was found to be an appropriate matrix and sodium trifluoroacetate was used as the cation source to generate sodium adduct of the polystyrene. MALDI-TOF MS data were calibrated against SpheriCal dendritic calibrants from Polymer Factory (Stockholm, Sweden). $\mathrm{M}_{\mathrm{n}}$ and $Đ$ of the resultant spectra were calculated using Polytools software. Data in Figure 2 and Figure S4 were collected from Bruker-Daltonics MALDI-TOF Autoflex Speed mass spectrometer in linear mode with positive ion detection. DCTB was used as MALDI matrix and NaTFA was used as cationization agent. Mass calibration was performed using homemade polystyrene standards. Typical sample preparation was performed by making stock solutions in THF of matrix $(20 \mathrm{mg} / \mathrm{ml})$, polymer analyte $(1 \mathrm{mg} / \mathrm{ml})$, and NaTFA $(2 \mathrm{mg} / \mathrm{ml})$. The stock solutions were mixed in a 100/1/1 ratio (matrix/analyte/cation), and deposited onto the MALDI target plate.

\begin{tabular}{|c|c|c|c|c|}
\hline & \multicolumn{2}{|c|}{ MALDI-TOF MS } & \multicolumn{2}{c|}{ SEC* } \\
\hline Polymer & $\mathbf{M}_{\mathbf{n}}$ & $\mathbf{Ð}$ & $\mathbf{M}_{\mathbf{n}}$ & Đ \\
\hline $\mathbf{2 a}$ & 2850 & 1.05 & 2500 & 1.12 \\
\hline $\mathbf{3 a}$ & 2700 & 1.01 & 2250 & 1.09 \\
\hline $\mathbf{2 b}$ & 3600 & 1.01 & 3800 & 1.14 \\
\hline $\mathbf{3 b}$ & 3600 & 1.02 & 3200 & 1.12 \\
\hline
\end{tabular}

Table S1: Mass determination of $l$-PS (2) and $c$-PS (3) by SEC and MALDI-TOF MS

*Mn values were measured by calibrations with PS standards. Cyclic $\mathrm{M}_{\mathrm{n}}$ were corrected based on $\mathrm{M}_{\mathrm{n} \text {-PS } \mathrm{PS}} / \mathrm{M}_{\mathrm{n} l \text {-PS }}$ observations based on SEC characterization using linear PS standards as reported by Alberty et al. ${ }^{2}$ ( 0.80 for $\mathrm{PS}_{3000}$ and 0.78 for $\mathrm{PS}_{3800}$ ). 


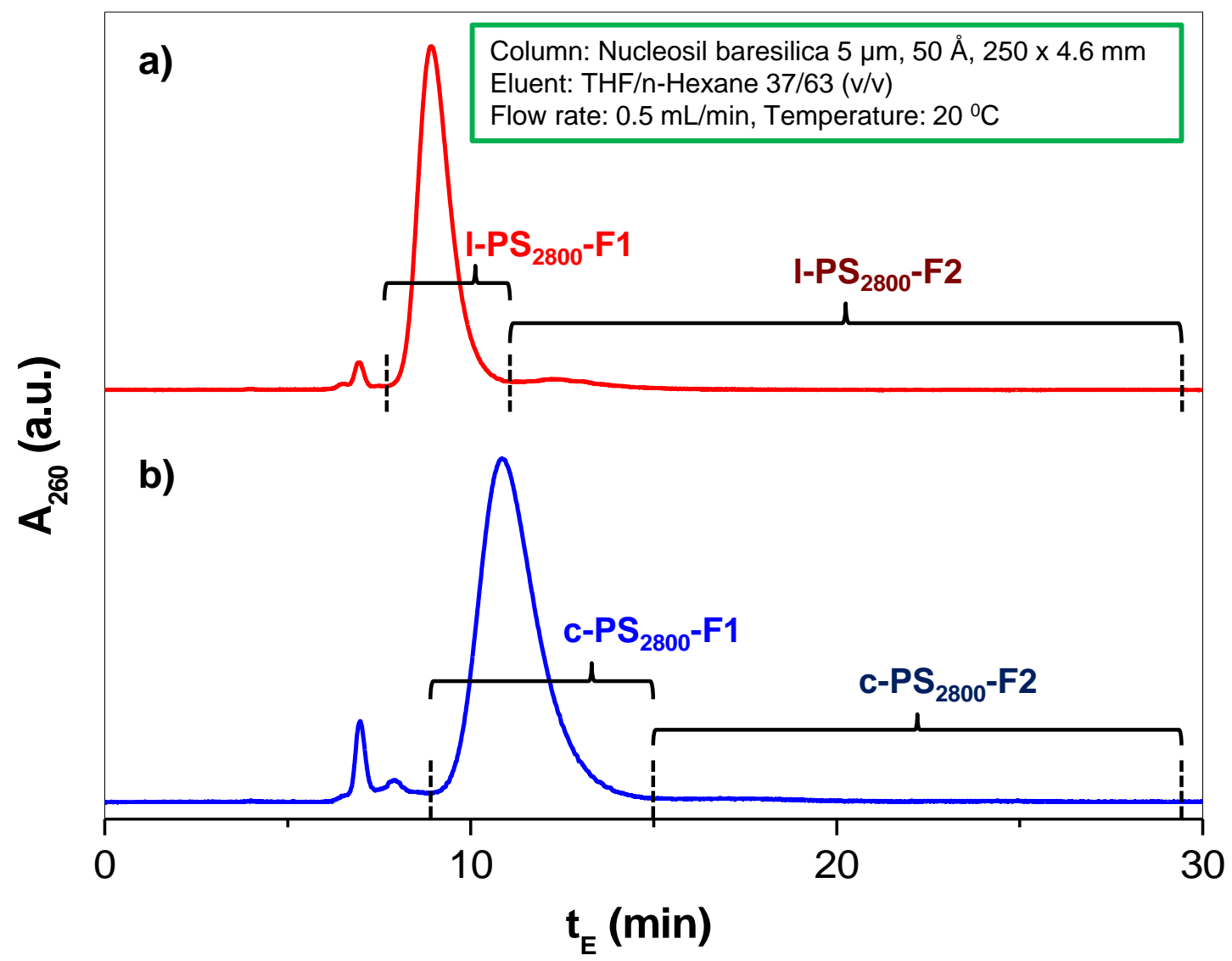

Figure S1: HPLC chromatograms during the separation and isolation of the unimer fraction 10 days after azidization and cyclization for a) $l-\mathrm{PS}_{2800}$ and b) $c-\mathrm{PS}_{2800}$ 


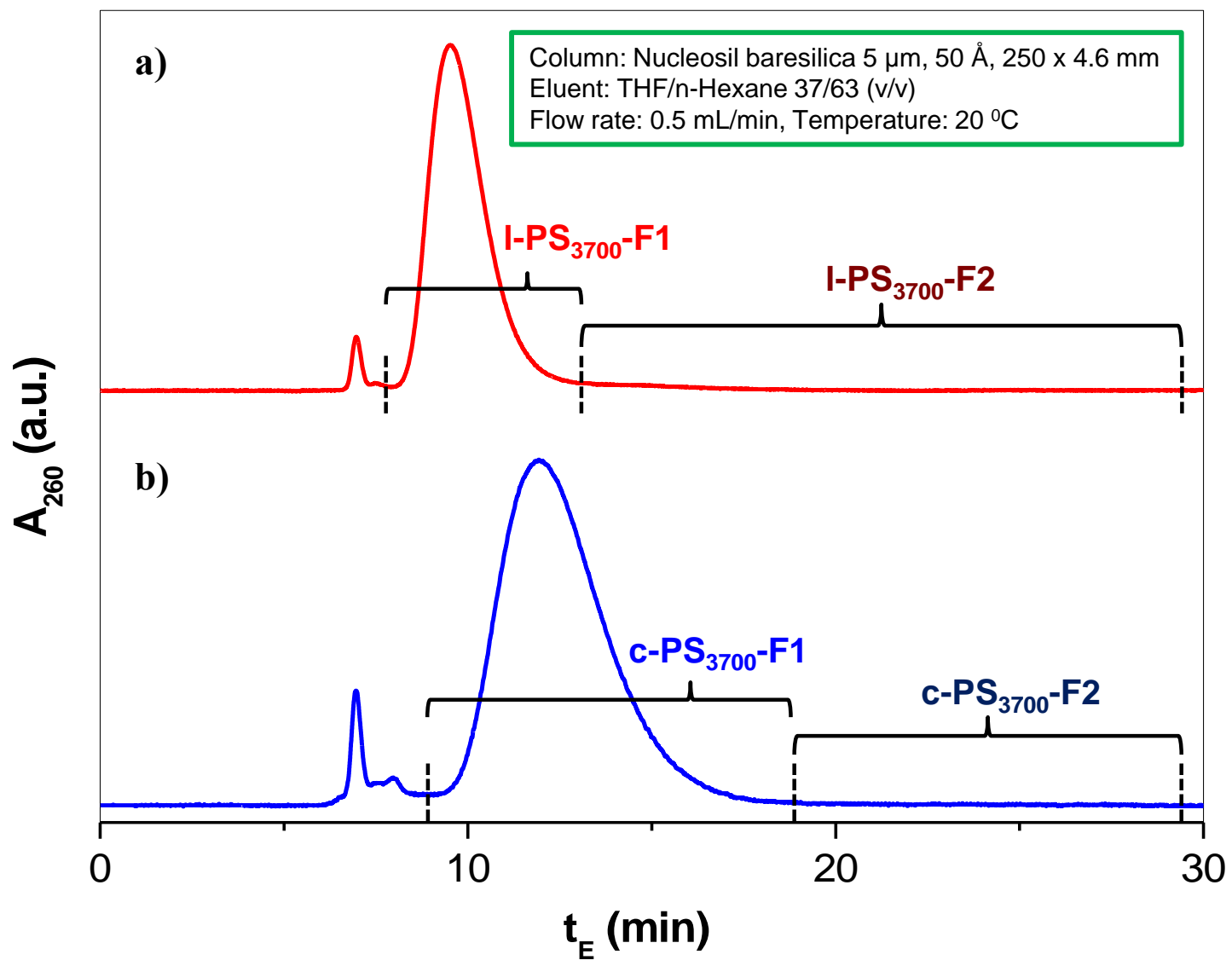

Figure S2: HPLC chromatograms during the separation and isolation of the unimer fraction 10 days after azidization and cyclization for a) $l$ - $\mathrm{PS}_{3700}$ and b) $c-\mathrm{PS}_{3700}$ 

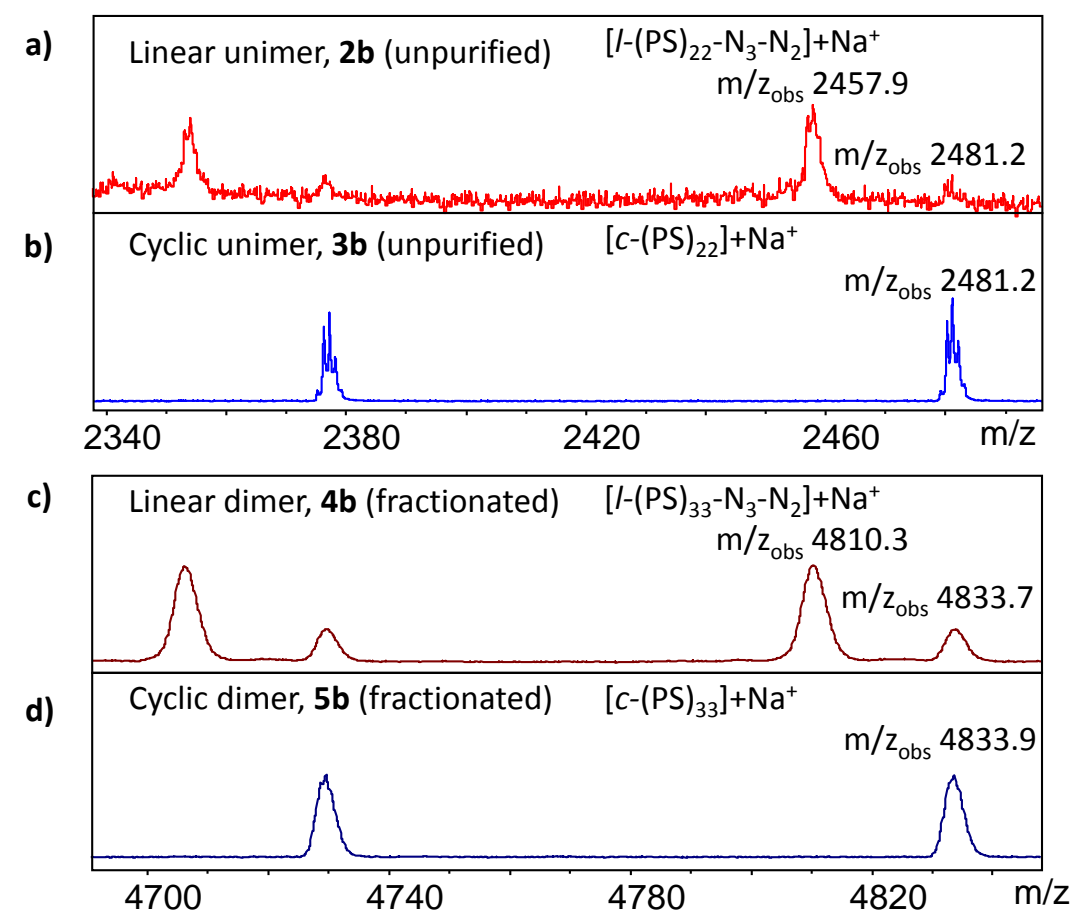

Figure S3: MALDI-TOF mass spectra in reflector mode of a) $l$-PS 2800, b) $c-\mathrm{PS}_{2800}$, c) $l d-\mathrm{PS}_{2800}$, d) $c d$-PS 2800 confirming the loss of the predominant metastable azide signal upon CuAAC cyclization to form the triazole linkage in the cyclic products. 

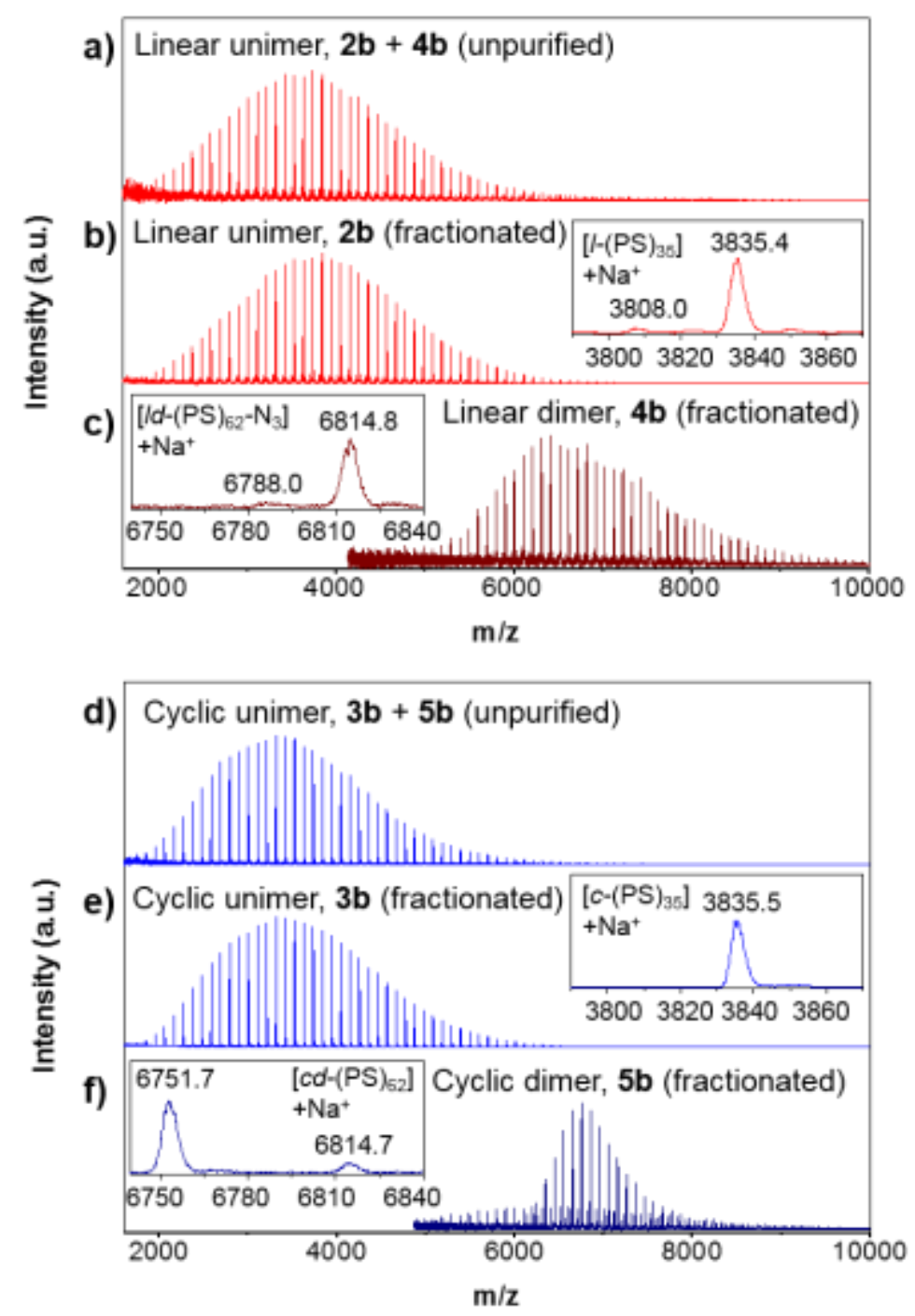

Figure S4: MALDI TOF mass spectra of a) $\left.\left.l-\mathrm{PS}_{3700,} \mathbf{2 b}+\mathbf{4 b}, \mathrm{b}\right) l-\mathrm{PS}_{3700}, \mathbf{2 b}, \mathrm{c}\right) l d-\mathrm{PS}_{3700}, \mathbf{4 b}$, and d) $c-\mathrm{PS}_{3700}, \mathbf{3 b}+\mathbf{5 b}$, e) $\left.c-\mathrm{PS}_{3700}, \mathbf{3 b}, \mathrm{f}\right) c d-\mathrm{PS}_{3700}, \mathbf{5 b}$ The theoretical $m / z$ for the $l$ - and $c$-PS 35 mer was 3835.5, the theoretical $\mathrm{m} / \mathrm{z}$ for the $l d$ - and $c d$-PS 62-mer was 6814.8. (Note: 6751.7 peak in $\mathrm{f}$ is cyclic unimer, $c$-PS 63-mer. A portion of the higher molecular weight unimer co-eluted with the trace amount of the dimer). 



Figure S5: MALDI-TOF mass spectra in reflector mode of a) $l$-PS 3700 , b) $c$-PS 3700 , a) $l d-\mathrm{PS}_{3700 \text {, }}$ b) $c d$-PS 3700 confirming the loss of the predominant metastable azide signal upon $\mathrm{CuAAC}$ cyclization to form the triazole linkage in the cyclic products. 


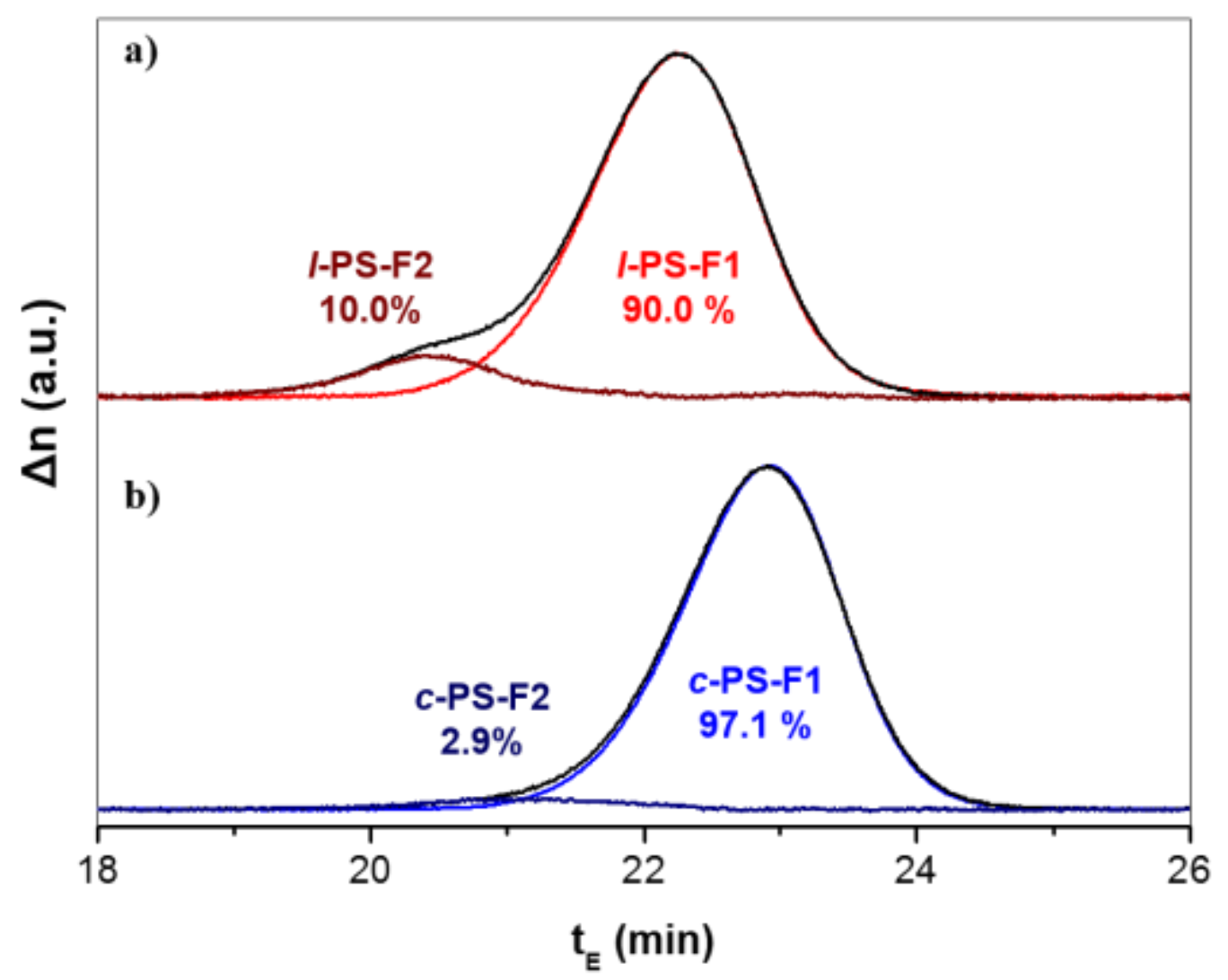

Figure S6: Representative SEC chromatograms of HPLC fractions 30 days after azidization and cyclization for a) $l-\mathrm{PS}_{2800}$ and b) $c-\mathrm{PS}_{2800}$. Fraction 1 corresponds to the isolated unimer fraction, while fraction 2 corresponds to the dimer fraction. 


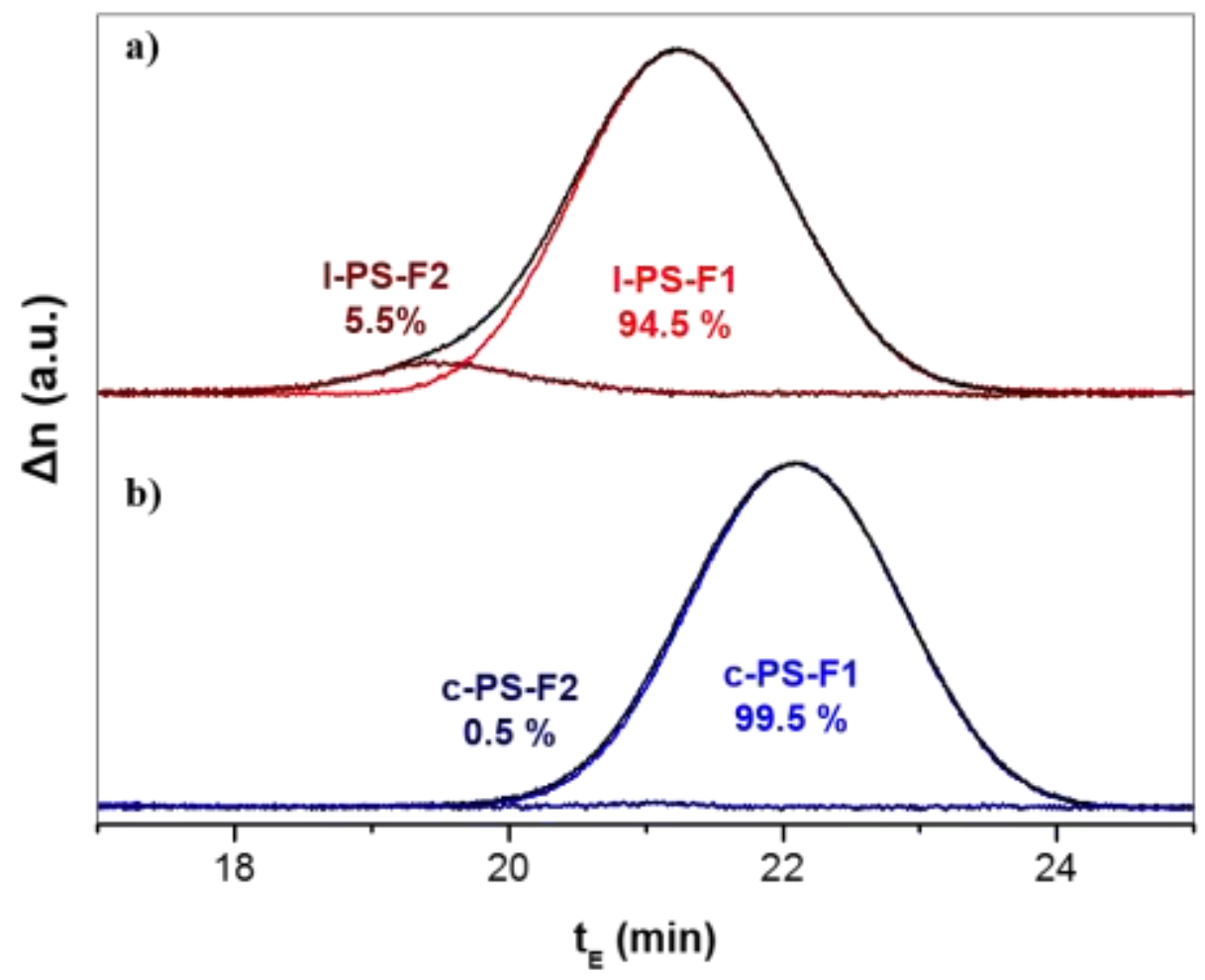

Figure S7: Representative SEC chromatograms of HPLC fractions 30 days after azidization and cyclization for a) $l$-PS 3700 and b) $c$-PS 3700 . Fraction 1 corresponds to the isolated unimer fraction, while fraction 2 corresponds to the dimer fraction. 




Figure S8: The SEC chromatograms of azidization reaction of 1a to generate $\mathbf{2 a}$ at $1 \mathrm{~h}, 4 \mathrm{~h}, 16$ $\mathrm{h}$ and $48 \mathrm{~h}$ show no appreciable sign of click oligomerization.

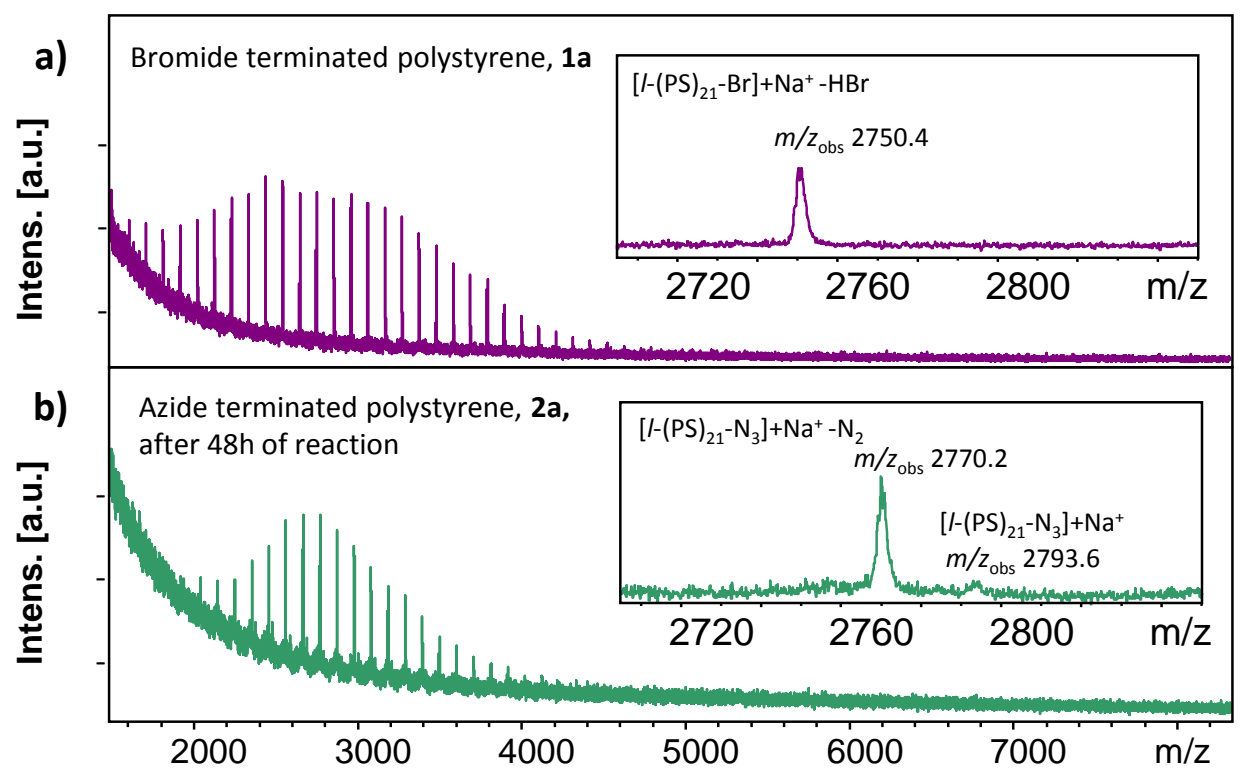

Figure S9: MALDI-TOF mass spectra of azidization reaction of 1a to generate 2a at a) $0 \mathrm{~h}$ where the major distribution corresponds to the sodium adduct of the $\mathrm{HBr}$ elimination product, ${ }^{3}$ $\left(\left[l-\mathrm{PS}{ }_{21}-\mathrm{Br}\right]+\mathrm{Na}^{+}-\mathrm{HBr} m / z_{\text {theo. }}=2750.9 \mathrm{~m} / \mathrm{z}_{\text {obs. }}=2750.4\right)$ and $\left.\mathrm{b}\right)$ at $48 \mathrm{~h}$ where the major distribution 
corresponds to the metastable azide signal ${ }^{4}\left(\left[l-\mathrm{PS}_{21}-\mathrm{N}_{3}\right]+\mathrm{Na}^{+}-\mathrm{N}_{2} m / z_{\text {theo. }}=2770.7 \mathrm{~m} / \mathrm{z}_{\text {obs. }}=2770.2\right)$ and the minor distribution corresponds to the parent azide signal $\left(\left[l-\mathrm{PS}_{21}-\mathrm{N}_{3}\right]+\mathrm{Na}^{+} \mathrm{m} / z_{\text {theo. }}=2794.0\right.$ $\left.m / z_{o b s .}=2793.6\right)$. The product exhibits no sign of click oligomerization.

1. Laurent, B. A.; Grayson, S. M. An Efficient Route to Well-Defined Macrocyclic Polymers via “Click” Cyclization. J. Am. Chem. Soc., 2006, 128, 4238-4239.

2. Alberty, K. A.; Hogen-Esch, T. E.; Carlotti, S. Synthesis and Characterization of Macrocyclic Vinyl-Aromatic Polymers. Molecular Weight-Dependent Glass Transition Temperatures and Emission of Macrocyclic Polystyrene. Macromol. Chem. Phys. 2005, 206, 1035-1042.

3. Li, Y.; Hoskins, J. N.; Sreerama, S. G.; Grayson, M. A.; Grayson, S. M. "The Identification of Synthetic Homopolymer End Groups and Verification of their Transformations Using MALDI-TOF Mass Spectrometry.” J. Mass Spectrom., 2010, 45, 587-611.

4. Li, Y.; Hoskins, J. N.; Sreerama, S. G.; Grayson S. M. MALDI-TOF MS Characterization of Polymers Containing an Azide Group: Evidence for Metastable Ions. Macromolecules, 2010, 41, $6225-6228$. 